\title{
GAIA: uma proposta de um guia de recomendações de acessibilidade de interfaces Web com foco em aspectos do Autismo
}

\author{
Talita C. P. Britto ${ }^{1}$, Ednaldo B. Pizzolato ${ }^{1}$ \\ ${ }^{1}$ Departamento de Computação - Universidade Federal do São Carlos (UFSCar) \\ Rodovia Washington Luis, km 235, CEP 13565-905 - São Carlos - SP-Brasil \\ contato@talitapagani.com, ednaldoddc.ufscar.br
}

\begin{abstract}
In this paper, we describe the proposal of an open-source and open access website designed to share a set of web accessibility guidelines for people with Autism Spectrum Disorder (ASD) called GAIA, that intends to help web developers to design accessible web interfaces for these users. The guidelines were extracted from a revision process of 17 works published between 2005 and 2015 including international recommendations, commercial or academic software and peer-reviewed papers. We identified 107 guidelines that were grouped in 10 categories through affinity diagram technique. Then, we normalized the guidelines in each group according to similarities and duplicated statements, generating a set of 28 guidelines. As a result, we evidenced best practices to design accessible web interfaces for people with ASD based on well succeeded solutions presented in works of different contexts. With those results, we aim to contribute to the state of the art of cognitive web accessibility. Therefore, we made the set of guidelines available in a repository on GitHub, so it can be used both by researchers and technical professionals.
\end{abstract}

Resumo. Neste artigo, descrevemos a proposta de um website de código e acesso abertos projetado para divulgar um conjunto de recomendações de acessibilidade web para pessoas com Transtorno do Espectro do Autismo (TEA) chamado GAIA, o qual tem a intenção de auxiliar desenvolvedores web a projetar interfaces web mais acessiveis a estes usuários. Estas recomendações foram extraídas através de um processo de revisão de 17 trabalhos publicados entre 2005 e 2015, incluindo recomendações internacionais, softwares comerciais ou acadêmicos e artigos revisados por pares. Identificamos 107 recomendações que foram agrupadas em 10 categorias através da técnica de diagrama de afinidades. Em seguida, normalizamos as recomendações em cada categoria de acordo com similaridades e declarações duplicadas, gerando um conjunto de 28 recomendações únicas. Como resultado, evidenciamos melhores práticas para projetar interfaces web acessiveis a pessoas com TEA baseado em soluções de sucesso presentes em trabalhos de diferentes contextos. Com estes resultados, esperamos contribuir com o estado da arte de acessibilidade web cognitiva. Dessa forma, disponibilizamos as recomendações em um repositório no GitHub, para que estes resultados possam ser utilizados tanto por pesquisadores quanto por profissionais técnicos. 
V Congresso Brasileiro de Informática na Educação (CBIE 2016)

Anais do XXVII Simpósio Brasileiro de Informática na Educação (SBIE 2016)

\section{Introdução}

O Transtorno do Espectro do Autismo (TEA) é uma síndrome dentro dos Transtornos Globais de Desenvolvimento (TGD) que afeta três aspectos do desenvolvimento humano: as habilidades sociais, de comunicação e de interesse [Ozand et al, 2003; CDPC, 2012; Brasil, 2013]. Pessoas com TEA podem apresentar dificuldades em interagir com outras pessoas, expressar-se verbal e não verbalmente, participar de brincadeiras de "faz-deconta" e imaginação, bem como apresentar um repertório restrito de interesses [Gadia, 2004; Brasil, 2013]. Quanto mais cedo o diagnóstico do TEA, mais efetivos podem ser os tratamentos para minimizar o impacto do comprometimento das habilidades e permitir melhor qualidade de vida às pessoas com autismo e seus familiares [Ozand et al, 2003; CDPC, 2012; Carrer et al, 2009; Brasil, 2013]. Estima-se que, no Brasil, 2 milhões de pessoas tenham manifestações do TEA [Oliveira, 2015].

Nos últimos 20 anos, tecnologias computacionais, especialmente relacionadas à hipermídia e Web, têm sido utilizadas como ferramentas de apoio para crianças com TEA, bem como para seus pais, terapeutas e educadores [Carrer et al, 2009; Battochi et al, 2010; Millen et al, 2010; Putnam e Chong, 2008]. Soluções de hipermídia educacional projetadas adequadamente para pessoas com TEA permitem que a tecnologia seja utilizada de forma benéfica para intervenção pedagógica ou terapêutica [Moore e Calvert, 2000; Millen et al, 2010; Millen et al, 2012; Muñoz et al, 2013; Silva et al, 2013]. Crianças com TEA usualmente têm uma alta preferência por uso de computadores e tablets.

Porém, o que se pode observar é que há uma lacuna de materiais que possam orientar os profissionais de TI a planejar e implementar soluções computacionais de hipermídia ajustadas às necessidades de pessoas com TEA [Mankoff et al, 2010; Millen et al, 2010; Millen et al, 2012]. É possível encontrar diversos websites e aplicativos educacionais voltados a pessoas com TEA, mas ainda desconhecemos quais soluções de design da interação e interface foram consideradas para desenvolver estes recursos e por quê, quais as barreiras de usabilidade e acessibilidade para pessoas com TEA foram ponderadas durante o desenvolvimento e quais estratégias de design se apresentaram mais eficientes para prover uma interação adequada.

Outro problema identificado é que muitas destas contribuições advindas de artigos científicos podem ter acesso restrito, expor uma linguagem de difícil compreensão para os profissionais da indústria ou apresentar uma carência de aplicabilidade prática das recomendações propostas.

Estes vieses motivaram o desenvolvimento do projeto intitulado Guia de

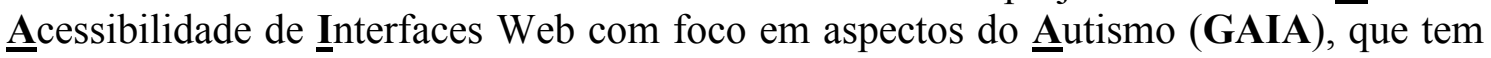
como objetivo prover um conjunto de diretrizes e recomendações de design para a acessibilidade com o intuito de apoiar desenvolvedores de softwares e educadores digitais a entender melhor como desenvolver websites mais adequados às necessidades de crianças com TEA, tendo como foco aplicações baseadas em plataforma Web e/ou dispositivos multitoque.

Neste artigo, descrevemos as principais características do website construído para a difusão das recomendações do GAIA baseado nos princípios de ciência aberta (opensource e open-access), bem como o processo de identificação, sistematização e consolidação das recomendações propostas. 
V Congresso Brasileiro de Informática na Educação (CBIE 2016)

Anais do XXVII Simpósio Brasileiro de Informática na Educação (SBIE 2016)

Este artigo está organizado no seguinte formato: na seção 2 apresentamos alguns trabalhos relacionados à investigação de recomendações de design para acessibilidade voltado à pessoas com TEA; na seção 3 apresentamos o método de pesquisa para a identificação das recomendações propostas; na seção 4 descrevemos a organização do website produzido para o GAIA e uma visão geral dos resultados obtidos para gerar as recomendações; por fim, na seção 5, sumarizamos as contribuições deste trabalho e apresentamos direcionamentos para trabalhos futuros.

\section{Trabalhos Relacionados}

Friedman e Bryan (2007) foram um dos primeiros autores a propor formalmente recomendações de acessibilidade web para pessoas com deficiências cognitivas, neuronais ou de aprendizagem, às quais iremos referir como CDNA a partir deste momento. Através de uma extensa revisão de literatura, os autores estabeleceram as 22 recomendações mais frequentes dentre os trabalhos analisados. De modo análogo, Darejeh e Singh (2013) investigaram princípios de usabilidade para pessoas com baixo letramento digital, incluindo pessoas com CDNA e especificamente pessoas com TEA.

Putnam e Chong (2008) conduziram em 2008 uma pesquisa (survey) com pais e educadores de crianças e adolescentes com TEA e diretamente com adultos com TEA. Os autores aplicaram um questionário online para identificar soluções de software que atendem às necessidades pedagógicas e terapêuticas de pessoas com TEA. O estudo não define recomendações de design, mas destaca aspectos que podem ajudar a construir produtos de tecnologia mais adequados à pessoas com TEA considerando seus objetivos, interesses e habilidades.

A Web Accessibility Initiative (WAI), um grupo de trabalho do World Wide Web Consortium (W3C) dedicado a estabelecer diretrizes de acessibilidade web, publicou em 2012 um rascunho de princípios de acessibilidade para pessoas com CDNA e suas respectivas barreiras de interação em potencial [Abou-Zahra, 2012]. Como estas recomendações tratavam-se de um rascunho, o W3C criou um grupo de força-tarefa intitulado Cognitive and Learning Disabilities Task Force (COGA) com foco na área agora denominada Acessibilidade Web Cognitiva. Em janeiro de 2015, a COGA publicou os resultados de uma pesquisa conduzida com usuários com CDNA para endereçar problemas e soluções de design para acessibilidade de conteúdos web, trazendo resultados significativos e ainda inéditos, embora necessitando uma investigação mais aprofundada [W3C, 2015].

De modo geral, a maioria dos trabalhos apresentam resultados preliminares e necessitam maiores detalhes para suas respectivas soluções propostas, apesar de apresentarem contribuições relevantes para a área. Um viés comum a todos os trabalhos é o foco em descrever as soluções para atender exclusivamente a lacuna de conhecimento de profissionais de tecnologia. Assim, estes trabalhos tendem a apresentar um conteúdo mais técnico. Recomendações que consideram as habilidades de pessoas com TEA e que podem ser utilizadas por profissionais de diferentes áreas têm um potencial de maior adoção e permite a realização de trabalhos multidisciplinares para desenvolver soluções mais adequadas não apenas no âmbito da funcionalidade, mas também quanto à objetivos pedagógicos e terapêuticos. 
V Congresso Brasileiro de Informática na Educação (CBIE 2016)

Anais do XXVII Simpósio Brasileiro de Informática na Educação (SBIE 2016)

\section{Métodos}

Para elaborar o conjunto de recomendações do GAIA, realizamos um processo dividido em três etapas: (1) seleção de contribuições, no qual realizamos uma revisão de literatura exploratória para realizar uma investigação mais flexível e menos estruturada que a revisão sistemática de literatura, uma vez que o estado da arte sobre acessibilidade web cognitiva ainda não é tão sólido quanto acessibilidade web em geral e optamos por um métodos que auxiliasse a definir uma visão geral acerca do tema e mapear problemas, limitações e relacionamentos entre conceitos apresentados em diferentes trabalhos; (2) extração, para extrair as recomendações potenciais dos trabalhos selecionados e realizamos agrupamento entre recomendações similares; (3) normalização $e$ consolidação, para conduzir um novo agrupamento entre as recomendações a fim de reduzir a granularidade das mesmas, realizando então a redação detalhada de cada recomendação e construindo, por fim, o website em plataforma de código aberto para disponibilizar as recomendações ao público. Detalhamos, a seguir, as atividades desenvolvidas em cada etapa.

Etapa 1 - Seleção dos estudos. Selecionamos trabalhos que abordavam acessibilidade de interfaces para pessoas com autismo ou outras deficiências cognitivas e neuronais. Para isso, analisamos: recomendações internacionais propostas pelo $\mathrm{W} 3 \mathrm{C}$ e WebAIM; três softwares e soluções assistivas; e trabalhos científicos revisados por pares publicados em conferências e periódicos nacionais e internacionais entre os anos $2005 \mathrm{e}$ 2015. Este processo de seleção envolveu uma pesquisa extensa em bases de dados como as bibliotecas virtuais da Association for Computer Machinery (ACM) e do Institute of Electrical and Electronics Engineers (IEEE), PubMed e Google Scholar. Também realizamos buscas manuais para encontrar soluções não restritas somente a artigos científicos.

Etapa 2 - Extração. Nesta etapa, realizamos uma primeira extração de recomendações de design dos trabalhos selecionados e realizamos o agrupamento de recomendações através da técnica de diagrama de atividades, gerado dez categorias (grupos) de recomendações. Em seguida, as recomendações que não puderam ser relacionadas a quaisquer grupos ou apresentavam conteúdo foram do escopo deste trabalho foram descartadas. Por fim, realizamos um novo agrupamento dentro de cada categoria unindo as recomendações duplicadas ou similares, gerando um esboço das recomendações.

Etapa 3 - Normalização e consolidação. Ao realizar o processo de redação detalhada das recomendações, objetivo desta etapa, identificamos algumas recomendações dentro de uma mesma categoria que possuíam sentenças altamente similares e poderiam ser concatenadas em uma única recomendação de modo a evitar redundâncias. Assim, realizamos um processo de normalização durante a consolidação das recomendações com o intuitivo de reduzir a granularidade das informações. Como forma de maximizar o uso de cada sentença identificada nos trabalhos durante a fase, adotamos a seguinte estratégia: o título de cada recomendação foi originado de um resumo das recomendações agrupadas; as sentenças similares foram utilizadas nas seções "Por que é importante para a pessoa com TEA" e "Como fazer" para manter a especificidade nos tópicos mais detalhados. Durante a redação, nos preocupamos em escrever o conteúdo de modo menos técnico possível, para que ele possa ser compreendido por profissionais e interessados que não sejam estritamente da área de computação. Após redigir as 28 
V Congresso Brasileiro de Informática na Educação (CBIE 2016)

Anais do XXVII Simpósio Brasileiro de Informática na Educação (SBIE 2016)

recomendações, construímos um website utilizando recursos simples de HTML e CSS, hospedado em uma plataforma de código aberto (GitHub), para que estes resultados sejam utilizados por diferentes profissionais em sua máxima extensão possível.

\section{Resultados}

Nesta seção, apresentamos um sumário dos resultados obtidos com a extração, normalização e sistematização das recomendações do GAIA e, nas próximas subseções, nos concentramos em descrever a estrutura do site desenvolvido para divulgar as recomendações do GAIA à comunidade brasileira de computação.

\subsection{Resumo dos trabalhos selecionados}

Ao selecionar os trabalhos a serem analisados no processo de revisão bibliográfica exploratória, consideramos os seguintes critérios: (1) artigos e guias de referência quanto à acessibilidade para pessoas com TEA ou deficiências cognitivas; (2) materiais que investigam e descrevem recomendações de acessibilidade para pessoas com deficiências cognitivas, neuronais ou de aprendizagem; (3) soluções computacionais, exceto de robótica, voltadas a pessoas com TEA, principalmente crianças, com evidências empíricas sobre a efetividade da solução onde seja possível extrair recomendações de design; (4) softwares e plataformas computacionais, comerciais ou acadêmicas, desenvolvidas para crianças com TEA; (5) recomendações que não sejam restritas a uma plataforma. Entre os 17 trabalhos analisados nesta revisão bibliográfica exploratória, três (3) são recomendações internacionais de acessibilidade web para pessoas com deficiência cognitiva, neuronal ou de aprendizagem; três (3) são softwares voltados para o ensino de pessoas com TEA ou outras deficiências cognitivas; um (1) trabalho é relacionado a recomendações de desenho universal para aprendizagem, independente do uso de tecnologia; e dez (10) trabalhos são artigos revisados por pares publicados em revistas ou anais de conferências científicas.

Apesar de a maioria das contribuições serem advindas de trabalhos dos Estados Unidos ( $47 \%$ dos trabalhos, $n=8)$, ainda foi possível trazer diversidade cultural com contribuições de outros oito países, incluindo trabalhos brasileiros. Com relação às plataformas de interação, selecionamos não apenas trabalhos relacionados à interfaces web, mas também contribuições de trabalhos sobre realidade virtual, mesa multitoque e aplicações nativas para desktop ou dispositivos móveis, devido à possibilidade de generaliza recomendações e padrões de interação que podem ser agnósticos quanto à plataforma. Grande parte dos trabalhos são relacionados a soluções de tecnologia voltados a pessoas com TEA, entretanto, as contribuições oriundas de organizações internacionais como o W3C e WebAIM não são exclusivamente focadas em aspectos do TEA.

Conforme descrito na Seção 3, as 107 potenciais recomendações que identificamos foram normalizadas em 28 recomendações únicas e distribuídas em 10 categorias, a saber: G01) Vocabulário Visual e Textual; G02) Customização; G03) Engajamento; G04) Representações Redundantes; G05) Multimídia; G06) Resposta às Ações; G07) Affordance; G08) Navegabilidade; G09) Estado do Sistema; G10) Interação com Tela Sensível ao Toque. Na Figura 1, apresentamos a distribuição das recomendações entre as categorias. 
V Congresso Brasileiro de Informática na Educação (CBIE 2016)

Anais do XXVII Simpósio Brasileiro de Informática na Educação (SBIE 2016)

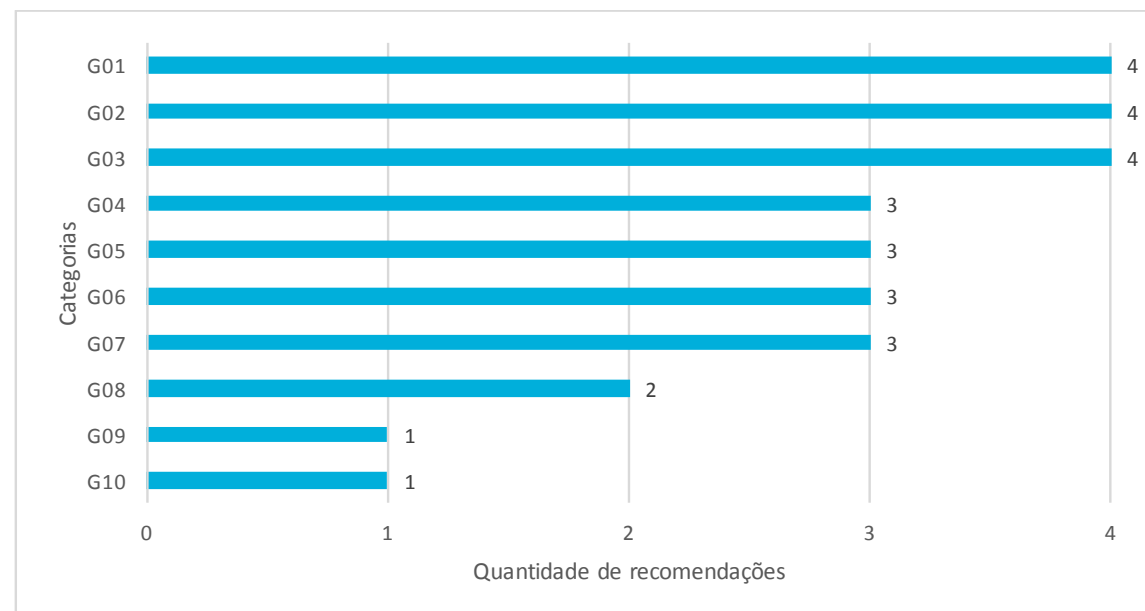

Figura 1. Distribuição das recomendações do GAIA entre as dez categorias.

\subsection{Página Inicial do site do GAIA}

O próprio conjunto de recomendações do GAIA era um entregáveis da dissertação de mestrado de um dos autores do artigo, porém, manter estas recomendações restritas à dissertação e à artigos científicos certamente reduzia a possibilidade utilização destes resultados de forma aplicada e prática. Dada a importância social deste material, a construção de um site era fundamental para propagar à comunidade os resultados e coletar feedbacks para melhoria contínua. Na Figura 2, apresentamos a página inicial do GAIA (http://talitapagani.com/gaia/), que conta com um layout simples, cores sóbrias e com contraste adequado entre texto e plano de fundo, apresentando o título do site à esquerda, opções de navegação e busca à direita e o conteúdo principal ao centro, sem elementos distrativos. A página inicial apresenta a listagem das recomendações cadastras em um formato de cartão (card), contendo o título curto da recomendação (ex.: Recomendação 10), a categoria a qual pertence, o título longo e tags que ajudam a classificar a recomendação. Destacamos que todas as recomendações possuem tags que associam a recomendação a uma característica do TEA ao qual ela pode potencialmente ser benéfica, como atenção, compreensão linguística, leitura, memorização, entre outros. O título, a categoria e as tags são links que levam à páginas específicas.

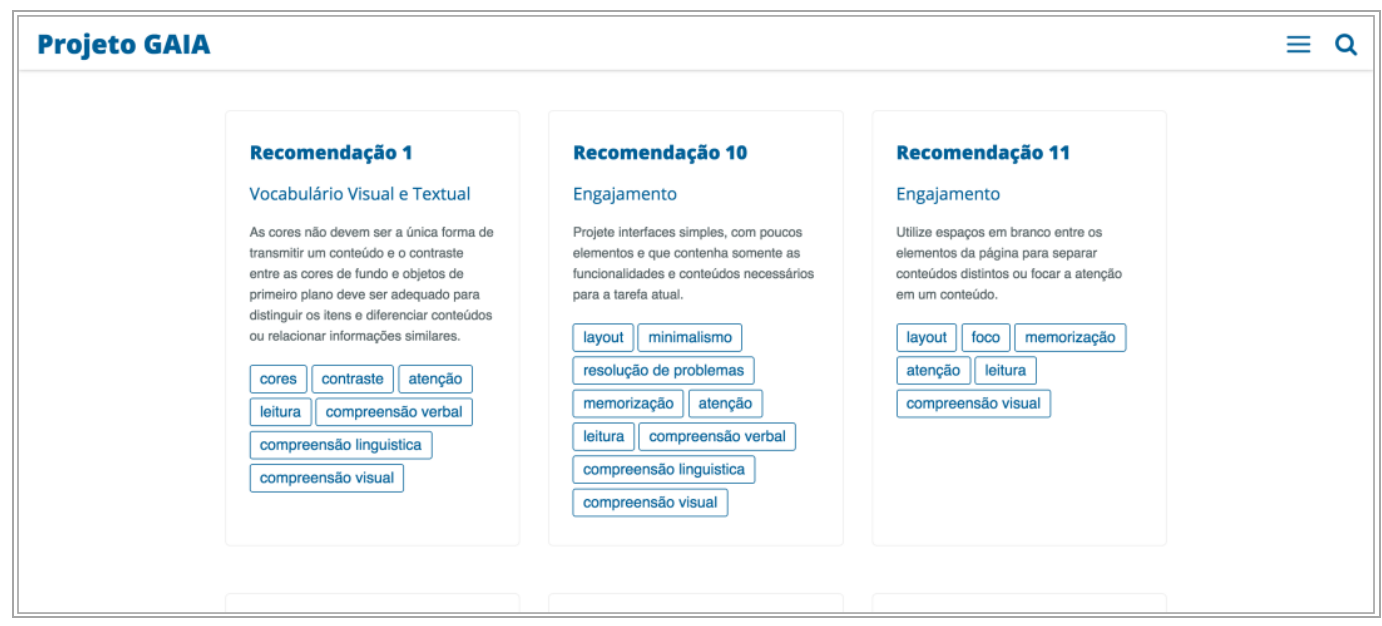

Figura 2. Página inicial do site do GAIA apresentando cartões (cards) com algumas recomendações. 
V Congresso Brasileiro de Informática na Educação (CBIE 2016)

Anais do XXVII Simpósio Brasileiro de Informática na Educação (SBIE 2016)

\subsection{Página de detalhe da recomendação}

Ao clicar no título de uma recomendação, o usuário é direcionado à página de detalha da mesma (Figura 3). Esta página também apresenta um layout focado no conteúdo principal e possui fontes maiores para aumentar a legibilidade do texto. No topo, na área com fundo azul a qual chamamos tecnicamente de hero, apresentamos a categoria da recomendação, o título curto (em maior destaque) e o título longo. Em seguida, apresentamos detalhes das recomendações que são diferenciais do GAIA: uma descrição mais aprofundada dos objetivos da recomendação; por que é importante atender a esta recomendação para a pessoa com TEA, ou seja, mesmo que ela se assemelhe a uma recomendação já estabelecida de acessibilidade web, qual o impacto específico no contex to do TEA; e uma lista de estratégias para auxiliar a implementar estas recomendações na prática. $\mathrm{O}$ próximo passo é incluir também imagens para exemplificar as recomendações.

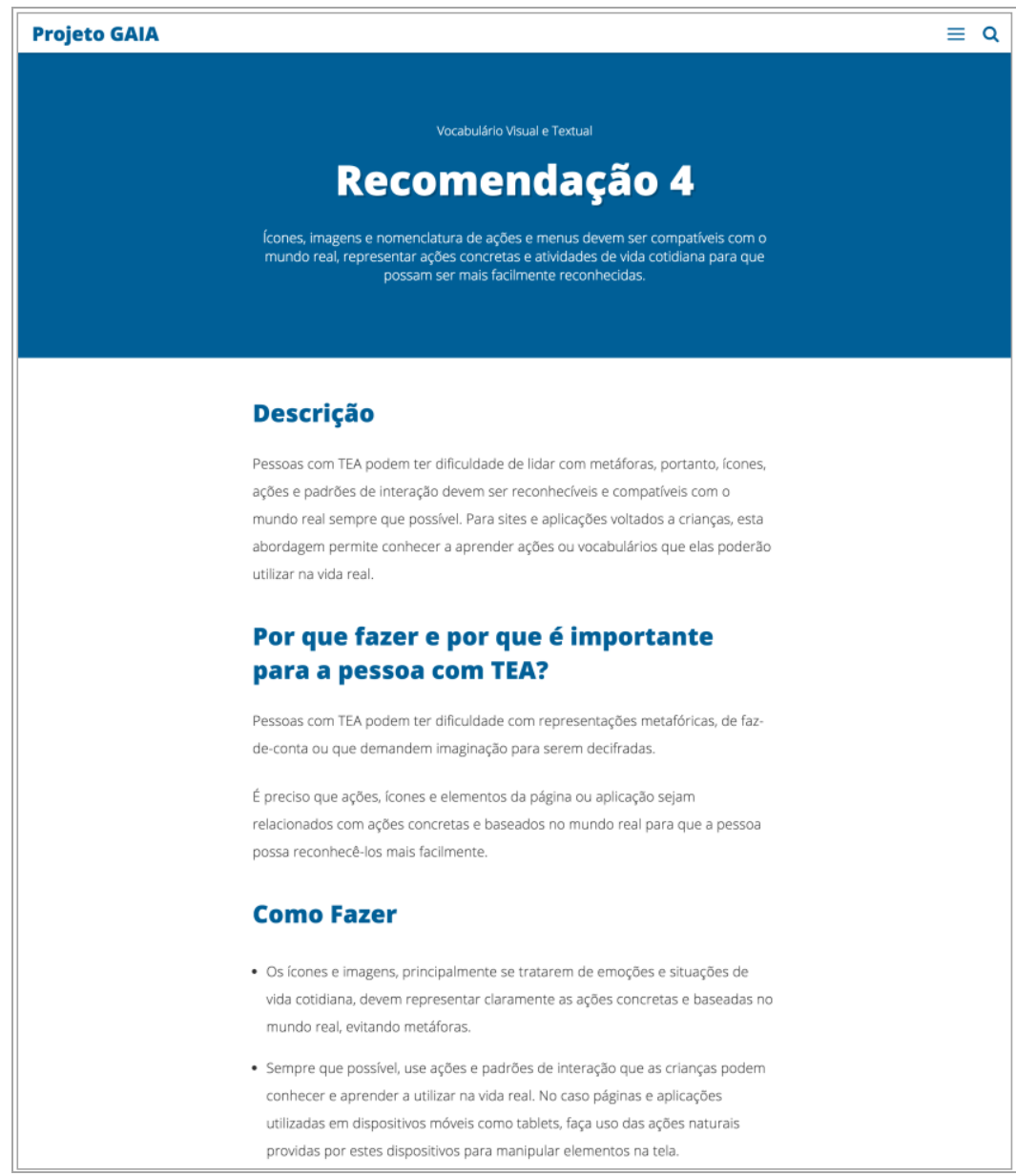

Figura 3. Página de detalhe de uma das recomendações, apresentando as informações de forma estruturada.

\subsection{Página de pesquisa por categorias}

Ao clicar no título de uma categoria, o usuário é redirecionado para uma página que apresenta a listagem de todas as recomendações organizadas por categoria (Figura 4). Esta página apresenta um layout similar à página de detalhe, mantendo uma consistência no modelo de interação. A página apresenta os títulos curto (como um link) e detalhado de cada recomendação. 
V Congresso Brasileiro de Informática na Educação (CBIE 2016)

Anais do XXVII Simpósio Brasileiro de Informática na Educação (SBIE 2016)

\subsection{Página de pesquisa por tags}

Ao clicar nas tags descritas no tópico 4.2., o usuário é direcionado para a página de Tags (Figura 5), onde as recomendações são divididas entre todas as tags já utilizadas para classificar as recomendações. Assim, uma recomendação pode aparecer repetidamente na página, uma vez que ela pode estar classificada em mais de uma tag. O layout desta página é simular à página de categorias. Exibimos na Figura 5 um trecho específico com rolagem de página com as recomendações que aparecem ao clicar na tag "memorização".

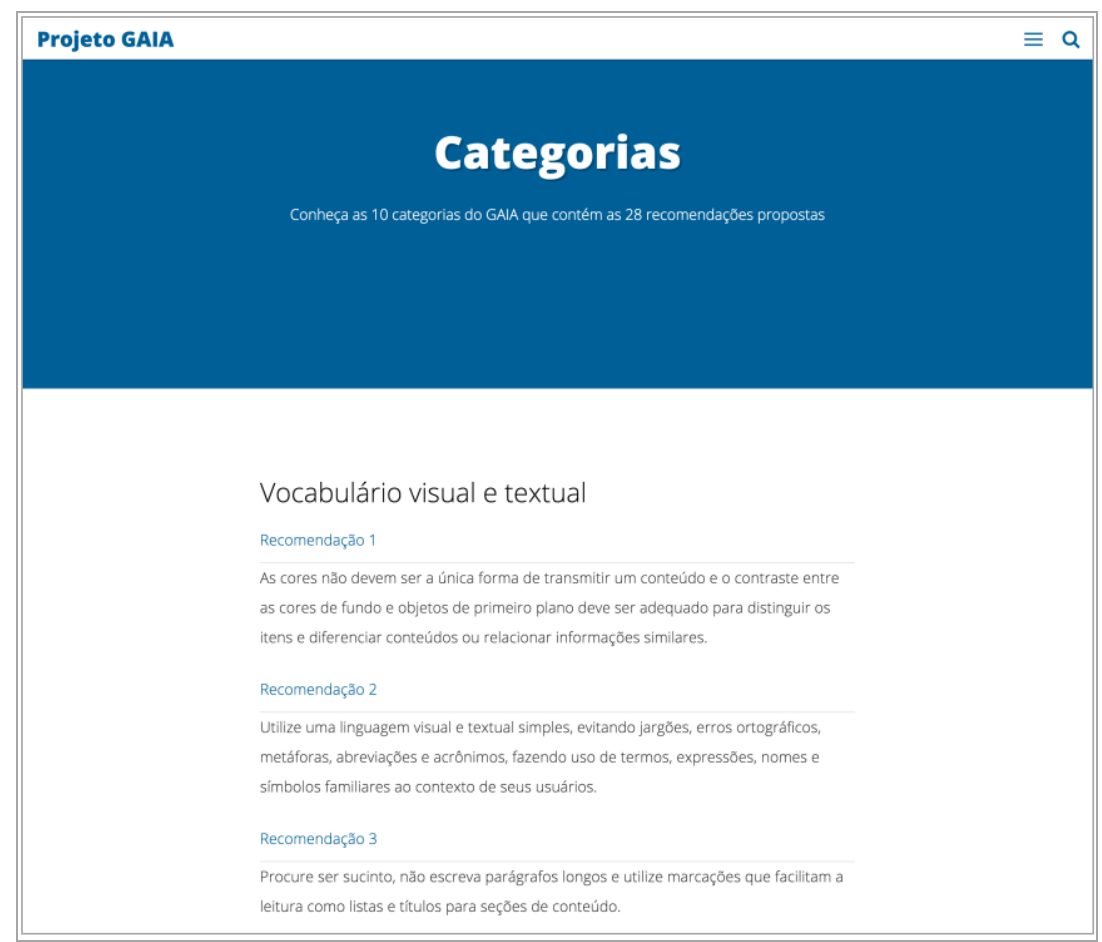

Figura 4. A página de categorias do GAIA apresenta o nome e o resumo de cada recomendação ordenados pelas categorias.

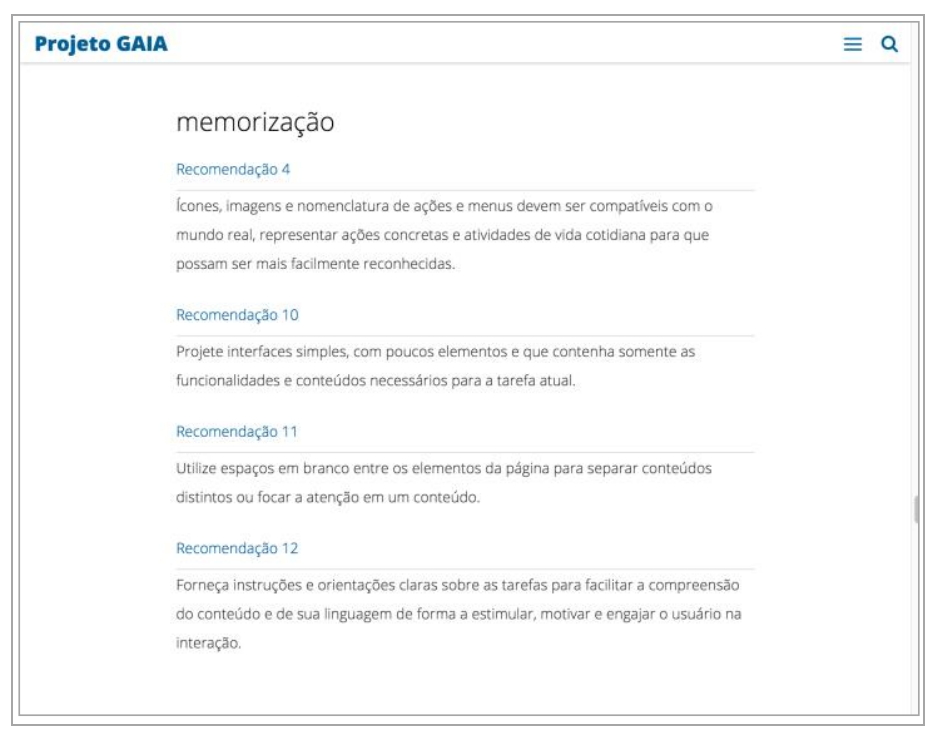

Figura 5. A página de tags apresenta as recomendações ordenadas através das tags. No topo da página, é possível ver a listagem de todas as tags. 
V Congresso Brasileiro de Informática na Educação (CBIE 2016)

Anais do XXVII Simpósio Brasileiro de Informática na Educação (SBIE 2016)

\subsection{Repositório do código-fonte do GAIA}

O projeto é hospedado no repositório de código aberto Github (http://github.com/talitapagani/gaia/). Nesta plataforma, comumente utilizada por programadores, é possível que qualquer pessoa que possua uma conta no sistema possa contribuir com o GAIA de diferentes formas: relatados problemas a serem corrigidos, cadastrando-os na aba Issues; fazendo uma cópia do projeto, corrigindo um problema/conteúdo e enviando esta contribuição aos autores (pull request) ou mesmo derivando este repositório (fork), seguindo as diretrizes de licenciamento. Seguir este princípio de ciência aberta possibilita um avanço maior dos resultados deste trabalho e torna-o mais inclusivo e participativo aos profissionais de tecnologia que podem ter contribuições relevantes e que podem tomar ciência de um projeto desenvolvido dentro da área acadêmica, fazendo também a ponte entre estas duas áreas que ainda se conversam pouco no Brasil. Disponibilizar este conteúdo em repositório de código aberto também possibilita a outras pessoas darem continuidade ao trabalho e não apenas ao pesquisador que desenvolveu a pesquisa originalmente.

\section{Considerações Finais}

Neste trabalho, descrevemos o processo de desenvolvimento de um conjunto de recomendações de design para a acessibilidade web, intitulado GAIA, focado em necessidades de pessoas com TEA. As recomendações propostas têm o intuito de evidenciar melhores práticas para desenvolver soluções acessíveis não somente a pessoas com TEA, mas também a outros públicos que podem ter as mesmas necessidades. Com a construção e difusão do GAIA através de um website de acesso aberto e de código aberto, esperamos que estas recomendações possam avançar na área de acessibilidade para pessoas com TEA como:

- Um material de apoio para que as pessoas consigam desenvolver websites e aplicativos adequados às necessidades de crianças com Autismo;

- Definições sobre o que funciona ou não para as pessoas com autismo quanto à utilização de tecnologias voltadas para Web;

- Um repositório de boas práticas e orientações para o projeto de interfaces Web com foco nas necessidades da pessoa com autismo que possa ser facilmente acessado, complementado, compreendido e distribuído;

- Um complemento aos materiais já existentes sobre acessibilidade web para pessoas com deficiência cognitiva, neuronal ou de aprendizagem adicionando técnicas relacionadas ao TEA.

A intenção de elaborar um website para o projeto GAIA partiu da preocupação em permitir que profissionais que não sejam da área de tecnologia, mas estejam envolvidos com produção de conteúdo digital para pessoas com TEA, possam compreender estas recomendações e entender melhor o contexto de uso da tecnologia pelas pessoas com TEA. O website pode ser um material útil até mesmo para os pais que queiram entender o que um aplicativo ou website para seus filhos deve atender para que seja engajador e de fácil utilização pela criança. O website do GAIA também é uma forma de apresentar as recomendações de forma prática, uma vez que ele vem sendo construído iterativamente aplicando as recomendações. 
V Congresso Brasileiro de Informática na Educação (CBIE 2016)

Anais do XXVII Simpósio Brasileiro de Informática na Educação (SBIE 2016)

\section{Referências}

Abou-Zahra, S. (ed). (2012). How People with Disabilities Use the Web. Status: Draft Updated 1 August 2012. http://www.w3.org/WAI/intro/people-use-web/diversity

Battochi, A. et al. Collaborative puzzle game: a tabletop interface for fostering collaborative skills in children with autism spectrum disorders. Journal of Assistive Technologies, v. 4, n. 1, 2010, p. 4-13.

Brasil. Ministério da Saúde. (2013). Diretrizes de Atenção à Reabilitação da Pessoa com Transtorno do Espectro do Autismo. Brasília: Ministério da Saúde.

Carrer, H.J., et al. (2009). Avaliação de software educativo com reconhecimento de fala em indivíduos com desenvolvimento normal e atraso de linguagem. Revista Brasileira de Informática na Educação, v. 17, n. 3, p. 1-16.

Centers for Disease Control and Prevention, CDCP. (2012). Prevalence of Autism Spectrum Disorders. MMRW, v. 61, n. 3, p. 1-19. http://www.cdc.gov/mmwr/pdf/ss/ss6103.pdf

Darejeh, A., Singh, D. (2013). A Review on User Interface Design Principles to Increase Software Usability for Users with Less Computer Literacy. In: Journal of Computer Science, v. 9, n. 11, Science Publications, 2013, p. 1443-1450.

Gadia, C.A. et al. (2004). Autism and pervasive developmental disorders. J. Pediatr., Porto Alegre, v. 80, n. 2.

Mankoff, J et al. (2010). Disability Studies as a Source of Critical Inquiry for the Field of Assistive Technology. In: Proceedings of the 12th international ACM SIGACCESS conference on Computers and accessibility (Assets '10), ACM, Orlando, Florida, USA, 2010.

Millen, L. et al. (2010). The Development of Educational Collaborative Virtual Environments for Children with Autism. In: Proceedings of the 5th Cambridge Workshop on Universal Access and Assistive Technology (CWUAAT), Cambridge, 2010.

Millen, L. et al. (2012). Collaborative virtual environment for conducting design sessions with students with autism spectrum conditions. In: Proceedings of International Conference of Disability, Virtual Reality and Associate Technologies, Laval, France, 2012, p. 269-278.

Moore, M., Calvert, S. (2000). Brief Report: Vocabulary Acquisition for Children with Autism: Teacher or Computer Instruction. Journal of Autism and Developmental Disorders, v. 30, n. 4, 2000, p. 359-362.

Muñoz, R., et al. (2012). Development of Software that Supports the Improvement of the Empathy in Children with Autism Spectrum Disorder. In: International Conference of the Chilean Computer Science Society.

Oliveira, C. (2015). Um retrato do autismo no Brasil. http://www.usp.br/espacoaberto/?materia=um-retrato-do-autismo-no-brasil

Ozand, P.T. et al. (2003). Autism: a review. Journal of Pediatric Neurology, [s.1.], v. 1, n. 2, p. $55-67$.

Putnam, C., Chong, L. (2008). Software and Technologies Designed for People with Autism: What do users want? In: Proceedings of the 10th international ACM SIGACCESS conference on Computers and accessibility (Assets '08). ACM, New York, NY, USA, p. 3-10.

Silva, G. F. M. et al. (2013). Metáforas de Perspectivas Culturais na (re) definição de padrões de colaboração de um jogo de multi-toque para usuários com autismo. In: Proceedings of IHC'13, Brazilian Symposium on Human Factors in Computing Systems, SBC, Manaus, AM, Brazil.

W3C. Cognitive User Research (2015). http://w3c.github.io/coga/user-research 